\title{
Multiple sclerosis: structural and functional integrity of the visual system following alemtuzumab therapy
}

\author{
Chenyu Wang (D) , ${ }^{1,2}$ Joshua Barton, ${ }^{2}$ Kain Kyle, ${ }^{1,2}$ Linda Ly, ${ }^{1}$ Yael Barnett, ${ }^{1,3}$ \\ Hans-Peter Hartung, ${ }^{2,4}$ Stephen W Reddel, ${ }^{2}$ Heidi Beadnall, ${ }^{2,5}$ Marinda Taha, ${ }^{2}$ \\ Alexander Klistorner, ${ }^{1,6}$ Michael Harry Barnett (1) 1,2,5
}

\begin{abstract}
- Additional supplemental material is published online only. To view, please visit the journal online (http://dx. doi.org/10.1136/jnnp-2021 326164)
\end{abstract}

For numbered affiliations see end of article.

\section{Correspondence to}

Dr Michael Harry Barnett, Brain and Mind Centre, The University of Sydney, Camperdown, NSW 2050,Australia; michael@ sydneyneurology.com.au

$\mathrm{CW}$ and $\mathrm{JB}$ are joint first authors.

AK and MHB are joint senior authors.

Received 22 January 2021 Accepted 2 June 2021 Published Online First 29 June 2021

\section{SLinked}

- http://dx.doi.org/10.1136/ jnnp-2021-326821

Check for updates

(C) Author(s) (or their employer(s)) 2021. No commercial re-use. See rights and permissions. Published by BMJ.

To cite: Wang C, Barton J,

Kyle K, et al. J Neurol

Neurosurg Psychiatry

2021:92:1319-1324.

\begin{abstract}
Objective To investigate potential neuroprotective and pro-remyelinating effects of alemtuzumab in multiple sclerosis (MS), using the visual pathway as a model.

Methods We monitored clinical, multifocal visual evoked potential (mfVEP) and MRI outcomes in 30 patients commencing alemtuzumab for relapsing MS, and a reference group of 20 healthy controls (HCS), over 24 months. Change in mfVEP latency was the primary endpoint; change in optic radiation (OR) lesion diffusion metrics and Mars letter contrast sensitivity over the course of the study were secondary endpoints.

Results In patients, we observed a mean shortening of mfVEP latency of $1.21 \mathrm{~ms}$ over the course of the study $(95 \% \mathrm{Cl} 0.21$ to $2.21, \mathrm{p}=0.013)$, not altered by correction for age, gender, disease duration or change in OR T2 lesion volume. Mean mfVEP latency in the HC group increased over the course of the study by 0.72 ms (not significant). Analysis of chronic OR T2 lesions (patients) showed an increase in normalised fractional anisotropy and axial diffusivity between baseline and 24 months (both $p<0.01$ ). Mean Mars letter contrast sensitivity was improved at 24 months vs baseline $(p<0.001)$, and driven by an early improvement, in both patients and $\mathrm{HC}$.

Conclusion We found evidence of partial lesion remyelination after alemtuzumab therapy, indicating either natural restoration in the context of a 'permissive' local milieu; or potentially an independent, pro-reparative mechanism of action. The visual system presents a unique opportunity to study function-structure specific effects of therapy and inform the design of future phase 2 MS remyelination trials.
\end{abstract}

\section{INTRODUCTION}

Multiple sclerosis (MS) is an inflammatory demyelinating disease of the central nervous system (CNS). Accrual of focal lesions and the development of grey and white matter atrophy correlate with the development of progressive neurological disability in most patients. ${ }^{12}$ MS disease-modifying drugs reduce the accumulation of lesions and brain atrophy, but generally do not result in continuing functional improvement in patients with established disability. Alemtuzumab, a humanised anti-CD52 monoclonal antibody approved for the treatment of relapsing MS, results in sustained reduction in active inflammatory disease and, in a proportion of treated patients, clinical improvement reflected in a progressive reduction in Expanded Disability Status Scale (EDSS) scores. ${ }^{3}$ While robust antiinflammatory effects of alemtuzumab may in part explain early clinical improvements, longer term benefits and sustained normalisation of brain atrophy rates raise the possibility of a neuroprotective mode of action and an effect, perhaps permissive, on restorative processes including remyelination. $^{45}$

Remyelination is prominent in early, relapsing $\mathrm{MS}^{67}$ and corresponds with functional recovery in animal models ${ }^{8}$ of demyelination. With increasing MS disease duration, there is failure of remyelination, ${ }^{9}$ rendering axons more vulnerable to physiological stress ${ }^{10}$ and subsequent neurodegeneration. Therefore, remyelination is potentially an important factor both for restoring function after relapse and for longer term neuroprotection; and therapeutics that impact CNS remyelination could be critical determinants of long-term outcomes in MS.

We hypothesised that sustained clinical improvements following alemtuzumab therapy will be reflected in partial restoration of electrophysiological and radiological measures that are abnormal in MS. In particular, evidence of remyelination of surviving axons within chronically demyelinated MS lesions would support the notion that the observed reduction in disability following alemtuzumab therapy cannot be explained by anti-inflammatory effects alone. While accelerated brain volume loss is an excellent candidate imaging biomarker of neurodegeneration, ${ }^{2} 1112$ this metric does not resolve axonal and myelin integrity, gliosis or oedema within brain white matter tracts; and novel methods are required to monitor disease progression, and response to therapy, with greater specificity. The visual system, which is highly susceptible to damage in MS, can be interrogated with a combination of advanced imaging techniques and visual electrophysiology to facilitate comprehensive structural and functional assessment of an entire sensory system. ${ }^{13}$

Optic neuritis $(\mathrm{ON})$ is the best developed model for testing the remyelinating potential of therapeutics. ${ }^{14}$ In this paradigm, latency of the visual evoked potential (VEP) is employed as a surrogate marker of myelin integrity/recovery in the optic nerve. However, since the incidence of $\mathrm{ON}$ is relatively low, adequately powered clinical trials require a large number of recruiting sites and a 
long recruitment window. On the other hand, optic radiation (OR) lesions are very common in MS, occurring in approximately $70 \%$ of patients with relapsing disease. ${ }^{15} 16$ Since the VEP signal is generated at the level of striate cortex, its delay reflects the degree of demyelination along the entire visual pathway, including the OR. As a result, recovery of the multifocal VEP (mfVEP) latency delay may potentially be used to monitor remyelination both within the optic nerve and the lesions of the OR. ${ }^{13}$

Advances in diffusion imaging-based tractography permit the OR to be separated/segmented from surrounding white matter; and lesions within the OR identified. ${ }^{17}$ While myelin itself cannot currently be directly and specifically imaged in lesions via MRI because its relaxation time is too short, several measures including magnetisation transfer ratio (MTR) and diffusion indices have been proposed as surrogate markers. ${ }^{18-20}$ We have previously explored the pathological substrates of diffusivity in coherent white matter tracts such as the OR. ${ }^{21}$ Using asymmetry analysis between lesional and non-lesional fibres, we showed that a lesion-restricted increase in radial diffusivity (RD) was related to myelin loss, providing a potential biomarker, at least in coherent fibre bundles, of remyelination. ${ }^{21} 22$ Therefore, we investigated the potential neuroprotective and pro-remyelinating effects of alemtuzumab using the visual pathway as a model.

\section{SUBJECTS AND METHODS}

The study was an investigator-sponsored study, funded by Sanofi-Genzyme (Protocol GZ-2015-11423).

\section{Subjects}

Thirty patients with relapsing MS (McDonald 2010 criteria), commencing alemtuzumab as prescribed by their treating physician, were enrolled after providing written, informed consent. Inclusion criteria included age $18-60$ years, active disease ( $\geq 1$ relapse and/or $\geq 1$ gadolinium-enhancing MRI lesion (GEL) in the previous 12 months), EDSS $0-5$ and disease duration $\leq 15$ years. Patients who had received treatment within 30 days prior to enrolment with steroids or intravenous immunoglobulins; were less than 6 months from the use of mitoxantrone, cyclophosphamide, azathioprine, methotrexate or mycophenolate; or had ever used of cladribine or alemtuzumab were excluded. In addition, 20 age-matched and sex-matched healthy controls (HCs) were enrolled as a reference group.

\section{Clinical and visual assessment}

Neurological assessments, including EDSS score (patients only) and low contrast visual acuity (Mars letter contrast sensitivity); mfVEP (VisonSearch, Sydney, Australia); and optical coherence tomography (OCT) were conducted at baseline, 6, 12 and 24 months. OCT scans were performed on non-dilated eyes with the same Cirrus HD-OCT device (Carl Zeiss Meditec AG, Jena, Germany) by qualified technicians. The Optic Disc Cube protocol was used to measure the peripapillary retinal nerve fibre layer (RNFL), with the average of all measures for each eye used in final analyses. Average mfVEP amplitude and latency were measured at each time point using TERRA software (VisionSearch, Sydney, Australia); and a latency progression analysis between baseline and 24 months calculated as the primary endpoint of the study using a modification of the method described by Klistorner et al. ${ }^{23}$ Briefly, for each segment (0-55) of each eye, the presence of appropriate VEP signal across baseline, 12 months and 24 months was assessed; waveforms from individual readable segments (ie, waveforms with detectable amplitude) were automatically mapped to the corresponding waveform from the time point with the largest amplitude using TERRA software. The required mean shift of all readable segment waveforms, measured between time points, was used to estimate mfVEP latency change over the course of the study. Individual eyes with clinical or electrophysiological (as defined by an mfVEP latency increase of $\geq 8 \mathrm{~ms}$ between successive time points) evidence of on-study $\mathrm{ON}$ were excluded from the final longitudinal analysis to facilitate the assessment of potential remyelination in chronic, inactive MS lesions. Post-hoc subanalysis of latency progression in patient eyes with mfVEP latency delay suggestive of demyelination $(>151 \mathrm{~ms})$, based on previously published data, ${ }^{24}$ was also performed.

\section{MRI protocol and analyses}

All scans were acquired at baseline, 6, 12 and 24 months on a 3T GE MR750 (General Electric, Milwaukee, Wisconsin, USA), using a multichannel head and neck (HDNV) coil; and included the following sequences: sagittal 3D fluid-attenuated inversionrecovery (FLAIR CUBE echo time (TE)/inversion time (TI)/ repetition time $(\mathrm{TR})=162 / 2181 / 8000 \mathrm{~ms}$; flip angle $=90$; echo train length $=24)$; pre-contrast and post-contrast (gadolinium) 3D high-resolution T1-weighted image (WI) using fast spoiled gradient echo (SPGR) with magnetisation-prepared inversion recovery pulse $(\mathrm{TE} / \mathrm{TI} / \mathrm{TR}=2.8 / 900 / 5.9 \mathrm{~ms}$, flip angle $=10)$; and axial diffusion weighted imaging (TE/TR $=82 / 8325 \mathrm{~ms}$ ) with a uniform gradient loading $\left(b=1000 \mathrm{~s} / \mathrm{mm}^{2}\right)$ in 64 directions (including an additional b0 image with reversed phase-encoding for distortion correction).

All analyses were performed by trained neuroimaging analysts at the Sydney Neuroimaging Analysis Centre. T2 lesion and GEL number and volumes were measured on FLAIR and T1 post-contrast images, respectively, using a semiautomated edge detection contouring technique. ${ }^{25}$ Subtraction imaging was performed to facilitate identification of new and enlarging T2 lesions at follow-up time points. Normalised volumetric analyses were performed at baseline using FMRIB's SIENAX software (V.2.6) ${ }^{26}$ following brain extraction and lesion-inpainting ${ }^{27}$ to correct for T1-hypointensity misclassification on T1-WI 3D SPGR images; and SIENA was used to calculate percentage brain volume change (PBVC) over course of the study.

Prior to calculation of lesion and diffusion metrics, the ORs were automatically demarcated on lesion-inpainted 3D T1 images at each time point using a non-linearly co-registered OR template. The method was chosen to best deal with the presence of lesions within the OR and to simplify the analysis framework. ${ }^{17}$ Baseline chronic T2 lesion masks were applied to all subsequent time points with non-linear co-registration to adjust for its shift related to brain atrophy. Active lesions (GELs) and their corresponding regions of interest (ROIs) detected at any time point were excluded on co-registered images from all other time points. Diffusion metrics were then calculated for each scan, including mean OR chronic lesion (for patients) and OR non-lesional (for patients and HCs) white matter mean diffusivity (MD), axial diffusivity (AD), RD and FA. Chronic lesion metrics were normalised to the subject's normal-appearing white matter (NAWM) at each time point (dLesion/dNAWM) to correct for intersubject and intrasubject variation. Proprietary software (K-WRAP, Sydney Neuroimaging Analysis Centre), which consolidates the above analysis pipelines in a secure, traceable manner, was used to analyse the final digital data obtained from all patients to determine per cent change in each diffusion tensor imaging metric for all scans from baseline to 24 months, constituting secondary endpoints for the study. 


\section{Statistical analyses}

All analyses were performed using SPSS V.26 (IBM) and a p value of $\leq 0.05$ used to determine significance. The potential impact of alemtuzumab on mfVEP latency over the course of the study was studied using a general linear model (analysis of variance) with three repetitions (baseline, 12 months, 24 months), corrected for Bonferroni multiple comparisons, with age, gender, disease duration and OR lesion volume change used as covariates. A similar analysis was undertaken for reference purposes in the HC cohort, with age and gender used as covariates. Paired sample $\mathrm{t}$-tests were used to compare individual metrics at baseline and follow-up time points. Differences between patient subgroups (ON and non-ON eyes) for individual metrics were performed with the Mann-Whitney U test.

\section{RESULTS}

Baseline clinical and visual pathway characteristics

Thirty patients with relapsing MS (diagnosed according to the McDonald 2010 criteria) and 20 HCs were enrolled in the study. Twenty-six of 30 patients had recently ceased treatment with another disease-modifying therapy, including fingolimod (10 of 30 ), natalizumab ( 8 of 30 ), dimethyl fumarate (4 of 30), glatiramer acetate (2 of 30) and interferon preparations (2 of 30$)$. Disease activity (13 of 26) and, for patients treated with natalizumab, JC virus antibody positivity ( 8 of 26 ) were the primary indications for the treating clinician's decision to commence alemtuzumab. Four patients with highly active disease were commenced on first-line therapy with alemtuzumab by their treating clinician.

Baseline demographic, clinical, mfVEP, OCT and conventional MRI metrics in the patient and HC cohorts are shown in table 1. As expected, the baseline mfVEP and OCT metrics in the MS and HC reference cohorts were discrepant. Less than half of the MS cohort had experienced a previous clinical episode of ON. Letter contrast sensitivity was normal in $38.0 \%$ of patient eyes and $78.9 \%$ of HC eyes. Conversely, moderate-severe deficits (score $\leq 1.48$ ) were present in $36.2 \%$ of patient eyes and $7.9 \%$ of $\mathrm{HC}$ eyes. Grouping of the patient data by a history of $\mathrm{ON}$ in the tested eye showed significantly worse letter contrast sensitivity $(p=0.017)$ and global RNFL thinning $(p=0.032)$ at baseline in $\mathrm{ON}$ versus non-ON eyes, but there was no significant difference in mfVEP latency $(\mathrm{p}=0.058)$ or amplitude $(\mathrm{p}=0.914)$. The ORs were involved to some degree by visible pathology on MRI in 29 of 30 patients (96.7\%), with a mean baseline chronic lesion volume of $0.66 \mathrm{~mL}$ and $0.61 \mathrm{~mL}$ in the left and right $\mathrm{ORs}$, respectively.

\section{Baseline conventional and advanced imaging characteristics}

Conventional imaging analysis showed a high burden of radiological disease activity in the patient cohort, with a mean GEL count (SD) of 5.7 (16.3), range 0-83. The overall mean brain T2 lesion number (SD) was 44.3 (31.7), range 2-165. Baseline whole brain and thalamus volumetric data for both patients and healthy controls are shown in table 1 . Values for all baseline diffusion metrics are included in the online supplemental table 1.

\section{Clinical outcomes}

Four patients and three controls either withdrew or were lost to follow-up over the 24-month follow-up period. All but one patient completed both year 1 and 2 courses of alemtuzumab. Six patients experienced a total of seven clinical relapses (one patient experienced two clinical relapses) during the course of the study. In one case, this was associated with the development of a tumefactive
Table 1 Baseline demographic, VEP, OCT and conventional MRI metrics

\begin{tabular}{|c|c|c|}
\hline Variable & Patients with MS & Healthy controls \\
\hline Mean age in years (SD) & $36.8(7.1)$ & $36.0(9.4)$ \\
\hline Age range (years) & $25.0-54.2$ & $22.2-55.4$ \\
\hline Male:female & $3: 7$ & $2: 3$ \\
\hline Disease phenotype* & RMS (100\%) & \\
\hline $\begin{array}{l}\text { Mean disease duration from diagnosis } \\
\text { (SD) (years) }\end{array}$ & $5.3(4.6)$ & \\
\hline $\begin{array}{l}\text { Mean disease duration from symptom } \\
\text { onset (SD) (years) }\end{array}$ & $7.1(4.8)$ & \\
\hline \multicolumn{3}{|l|}{ Clinical relapses (SD), median (range) } \\
\hline Previous 12 months & $1.2(1.2), 1(0-5)$ & \\
\hline Previous 24 months & $2.0(2.0), 2(0-8)$ & \\
\hline Total previous relapses & $4.8(2.8), 4(1-10)$ & \\
\hline \multicolumn{3}{|l|}{ Previous optic neuritis (ON) (\%) } \\
\hline Yes & $12 / 30(40)$ & \\
\hline No & $17 / 30(57)$ & \\
\hline Unknown & $1 / 30(3)$ & \\
\hline Mean EDSS (SD) & $2.8(1.4)$ & \\
\hline EDSS range & $0.0-6.0$ & \\
\hline $\begin{array}{l}\text { Mean Mars letter contrast sensitivity } \\
\text { score }\end{array}$ & 1.63 & 1.76 \\
\hline Mean mfVEP latency (SD) (ms) & $161.72(14.09)$ & $149.33(8.44)$ \\
\hline $\begin{array}{l}\text { Mean mfVEP latency in ON eyes (SD) } \\
\text { (ms) }\end{array}$ & $168.54(17.15)$ & \\
\hline $\begin{array}{l}\text { Mean mfVEP latency in non-ON eyes } \\
\text { (SD) (ms) }\end{array}$ & $159.83(14.141)$ & \\
\hline Mean global RNFL thickness $(S D)(\mu \mathrm{m})$ & $84.19(9.18)$ & $91.77(7.78)$ \\
\hline $\begin{array}{l}\text { Mean RNFL thickness in ON eyes (SD) } \\
(\mu \mathrm{m})\end{array}$ & $78.23(11.17)$ & \\
\hline $\begin{array}{l}\text { Mean RNFL thickness in non-ON eyes } \\
(\mathrm{SD})(\mu \mathrm{m})\end{array}$ & $85.98(8.82)$ & \\
\hline $\begin{array}{l}\text { Mean normalised thalamic volume total } \\
\text { (SD) (mL) }\end{array}$ & $18.67(2.33)$ & $20.85(1.25)$ \\
\hline Mean normalised brain volume (SD) (mL) & $1494.74(88.00)$ & $1543.58(66.23)$ \\
\hline Normalised brain volume range $(\mathrm{mL})$ & $1251.95-1653.18$ & $1435.89-1696.58$ \\
\hline Mean T2 lesion numbert (SD) & $44.3(31.7)$ & \\
\hline Mean T2 lesion volumet (SD) (mL) & $11.08(8.81)$ & \\
\hline Mean gad lesion number (SD), range & $5.7(16.3), 0-83$ & \\
\hline Mean gad lesion volume (SD) (mL) & $0.44(1.29)$ & \\
\hline $\begin{array}{l}\text { Mean OR T2 chronic lesion volume (SD) } \\
(\mathrm{mL})\end{array}$ & $\begin{array}{l}\text { R: } 0.61(0.52) \\
\text { L: } 0.66(0.51)\end{array}$ & \\
\hline
\end{tabular}

${ }^{*}$ McDonald 2010 criteria.

tExcludes lesions $<3 \mathrm{~mm}^{3}$.

EDSS, Expanded Disability Status Scale; gad, gadolinium; mfVEP, multifocal VEP; MS, multiple sclerosis; OCT, optical coherence tomography; OR, optic radiation; RMS, relapsing MS; RNFL, retinal nerve fibre layer; VEP, visual evoked potential.

MS lesion that occurred approximately 4 months after the first course of alemtuzumab, described previously. ${ }^{28}$ The mean annualised relapse rate (SD) fell from 1.24 (1.18) in the 12 months prior to study commencement (historical) to 0.12 (SD 0.25 ) over the 24 months of the study $(\mathrm{p}=0.000)$, representing a $>90 \%$ reduction. The mean EDSS score decreased from 2.88 (1.39) at baseline to $2.24(1.53)$ at 24 months $(p=0.116)$. Only the sensory Functional System Score change between baseline and follow-up reached significance (mean change $-0.54, \mathrm{p}=0.050$ ), with no significant change in other standard EDSS subcomponents.

\section{Visual system outcomes}

Two of 60 patient eyes and 0 of $40 \mathrm{HC}$ eyes were excluded from longitudinal analysis of visual outcomes based on the 
MEAN LATENCY CHANGE (BL to 24 MONTHS)

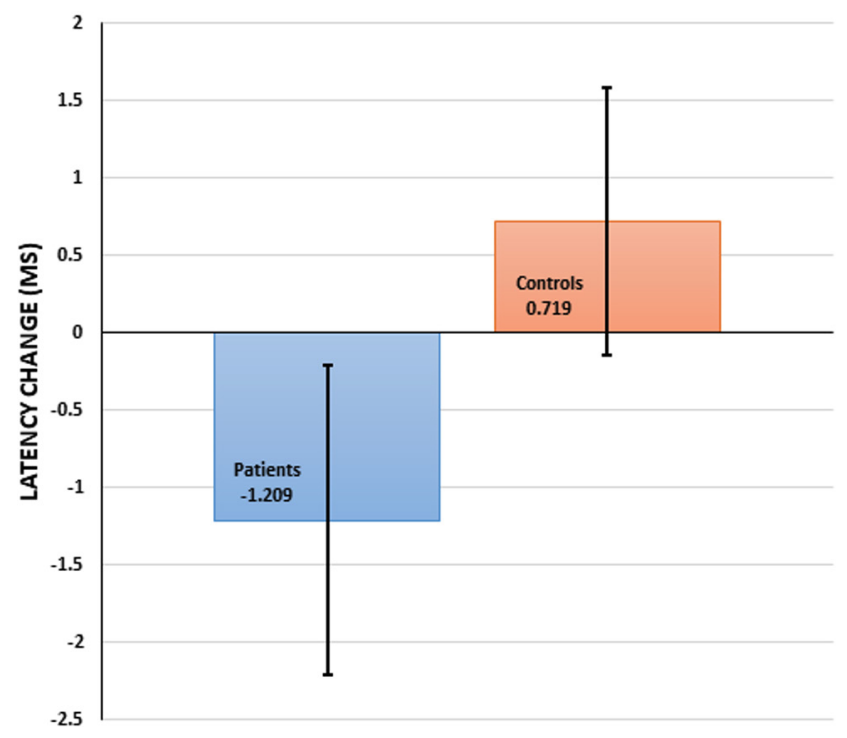

Figure 1 Mean latency change in patients and healthy controls. BL, baseline.

development of ON during the study. In patients, the mean Mars average letter contrast sensitivity was improved at 24 months vs baseline $(\mathrm{p}<0.001)$, however this difference appeared to be driven by a significant early (baseline to 6 months) improvement, with no change in the latter 12 months of the study; an identical pattern of apparent improvement was observed in the HC group. Progression analysis showed a mean shortening of mfVEP latency of $1.21 \mathrm{~ms}$ over the course of the study $(95 \% \mathrm{CI}$ 0.21 to $2.21, \mathrm{p}=0.013$ ), which was not altered by correction for age, gender, disease duration or change in OR T2 lesion volume (figure 1). Mean mfVEP latency in the HC group increased over the course of the study by $0.72 \mathrm{~ms}$, but this change was not significant. Post-hoc analysis following exclusion of patient eyes with mfVEP latency $<152 \mathrm{~ms}$ showed a slightly more robust shortening of mfVEP latency $(-1.44 \mathrm{~ms}, 95 \%$ CI 0.39 to 2.48 , $\mathrm{p}=0.004$ ) following alemtuzumab therapy. There were no significant changes in mfVEP amplitude over 24 months. There was a marginal increase $(1.42 \mu \mathrm{m})$ in global RNFL thickness in patients $(p=0.034)$ that remained significant after correction for age, gender and disease duration; no change in RNFL thickness was observed in controls.

\section{Imaging outcomes}

In patients, the mean number (SD) of whole brain GELs at baseline $(5.7(17.2))$ decreased by $81 \%(\mathrm{p}=0.164)$ at 24 months (1.1 (3.9)). The data were, however, skewed by the inclusion of a patient (patient 25) who responded poorly to alemtuzumab (based on MRI appearances), with 20 GELs on the 24-month scan (range among all other patients $=0-3$ ). There was no significant difference in whole brain T2 lesion number or volume between baseline and 24 months. Assessed cumulatively, no MRI activity (new T2 or enlarging T2 lesions; or GELs) was observed in 11 of 30 patients at any point in the study. Six GELs were seen in patient ORs across all study time points. There was a non-significant increase in the mean OR total T2 lesion volume $(0.03 \mathrm{~mL})$ between baseline and 24 months. Annualised PBVC in patients over the course (baseline vs 24 months) of the study was $-0.86 \%$. However, annualised PBVC between baseline and 6 months was $-1.31 \%$, raising the possibility that accelerated atrophy/pseudoatrophy contributed in the 6 months after commencement of alemtuzumab. In HCs, annualised PBVC was $-0.22 \%$ over the course of the study and was similar $(-0.20 \%)$ between baseline and 6 months. Similarly, total absolute thalamus volume was reduced by $0.24 \mathrm{~mL}$ at 24 months compared with baseline $(\mathrm{p}<0.001)$ in patients, but remained stable in controls.

Advanced imaging analysis of chronic OR T2 lesions showed a significant increase in normalised FA and AD between baseline and 24 months (both $\mathrm{p}<0.01$ ), and a non-significant reduction in normalised RD. Details for advanced imaging metrics are included in the online supplemental figure 1 and online supplemental table 2 .

\section{DISCUSSION}

Irreparable brain tissue loss occurs early in MS and continues throughout the disease course, contributing to the accrual of cognitive, motor and other functional deficits. Alemtuzumab has a profound anti-inflammatory effect in most treated patients, resulting in sustained reductions in relapse rate and MRI activity over at least 9 years. ${ }^{29}$ Here we exploited the visual system, which is highly susceptible to damage in MS, with combination of advanced imaging techniques and visual electrophysiology, to resolve changes in brain myelin and axonal structure and function following treatment with alemtuzumab. A modest but significant shortening of mfVEP latency $(-1.21 \mathrm{~ms})$ in patients was observed over 24 months, potentially reflecting remyelination of MS lesions in the visual pathway.

Similar, modest improvements in VEP latency have recently been reported after use of clemastine fumarate ${ }^{30}$ and opicinumab $^{14}$ in patients with relapsing MS. Preclinical data for both of these agents demonstrate effects on oligodendrocyte precursor cells and robust remyelination in the absence of immunomodulation. ${ }^{31}$ Alemtuzumab, a humanised anti-CD52 monoclonal antibody, results in profound immune cell depletion and longer term restoration of dysregulated immune networks ${ }^{42}$; in keeping with pivotal clinical trials of alemtuzumab, both clinical and MRI inflammatory activities were largely suppressed in our patient cohort. The present study therefore does not permit dissociation of this anti-inflammatory effect from a potentially independent, pro-reparative mechanism of action. Apparent lesion repair after alemtuzumab therapy may therefore simply represent natural restoration in the context of a 'permissive' local milieu. Interestingly, our study showed a marginal increase in global RNFL in patients with MS over 2 years, which we interpret as evidence of axonal stability in the optic nerve, which in turn could potentially reflect remyelination with secondary neuroprotection.

We observed a consistent increase in AD (and FA) in both whole brain and OR chronic lesions, with stability of $\mathrm{RD}$ and MD over 2 years. Previous studies have shown a progressive increase in MD in chronic MS lesions, reflecting ongoing axonal attrition. ${ }^{33}$ The expected rise in RD in this context may have been counterbalanced by the effect of remyelination following treatment with alemtuzumab in the current study. The capacity of chronic lesions to remyelinate is dependent on the number of surviving axons they contain; and restricting the analysis to lesions with sufficient axonal preservation using baseline $\mathrm{MD}$, MTR or even T1-hypointensity may have increased the power to more robustly detect evidence of remyelination.

Translation of electrophysiological and MRI biomarkers of remyelination into measurable clinical outcomes, particularly in small studies of this duration, is problematic. While Mars letter 
contrast sensitivity was considerably improved at 24 months vs baseline, improvement occurred at 6 months and was reflected in a similar pattern of improvement in controls, suggesting a learning effect. The benefits of remyelination are probably underpinned by reduced axonal attrition in chronically demyelinated lesions; and overt clinical benefits may therefore only be appreciable in longer term placebo-controlled studies.

While the visual system presents a unique opportunity to study function-structure specific effects of therapy, our observational study does highlight a number of considerations relevant to future phase 2 remyelination trials. For the main analysis, we did not exclude patients/eyes with normal mfVEP latency $(<152$ $\mathrm{ms}$ ), who in all likelihood have normal visual pathway conduction and therefore reduced the power of the study to show an effect on remyelination. Post-hoc analysis following exclusion of patient eyes with mfVEP latency $<152 \mathrm{~ms}$ showed a slightly more robust shortening of mfVEP latency $(-1.44 \mathrm{~ms}$, 95\% CI 0.39 to $2.48, p=0.004$ ) following alemtuzumab therapy. Second, the development of on-study new visual pathway pathology confounds the analysis of mfVEP latency as a measure of remyelination in chronic lesions; and exclusion of, or statistical correction for, affected eyes (based on clinical or VEP criteria) should be prespecified in the study plan. In our study, we excluded 2 of 60 eyes that met the prespecified analysis exclusion criterion of an abrupt (between successive time points) increase in mfVEP latency of $\geq 8 \mathrm{~ms}$, thought to most likely reflect the development of on-study ON. Similarly, we controlled for the development of new OR pathology on MRI, which may have an impact on mfVEP latency, though this did not alter the result. Our study was not sufficiently powered to detect changes in either mfVEP latency or MRI in specific subgroups, such as patients with a history of ON.

MRI outcomes in remyelination studies have focused on change in a variety of whole brain metrics, including MTR, diffusion indices and novel sequences such as myelin water imaging. ${ }^{1934}$ Although the myelin specificity of these techniques has improved, remyelination is less likely to be apparent in NAWM, and measurement should be focused on microstructural change within white matter MS lesions, which in our study accounted for only $0.1 \%$ of the whole brain volume. The assessment of chronic lesion remyelination is also confounded by the development of on-study whole brain MRI activity (new lesions or GELs); and relevant ROIs should therefore be excluded from the overall analysis. Lack of MTR or more advanced analyses, such as magnetisation transfer saturation imaging, ${ }^{35}$ myelin water imaging $^{36}$ or myelin mapping with T1 and T2 images ${ }^{37}$ is a limitation of our study; advances in image acquisition, including quantitative synthetic MRI, ${ }^{38}$ will permit the future inclusion of myelin imaging within clinically feasible acquisition time frames.

Although limited by its observational nature, relatively small size and lack of a true control group, our study nevertheless provides insights into the mechanisms of sustained clinical and MRI improvements following therapy with alemtuzumab and a framework for future remyelination trials.

\footnotetext{
Author affiliations

'Sydney Neuroimaging Analysis Centre, Camperdown, New South Wales, Australia ${ }^{2}$ Brain and Mind Centre, The University of Sydney, Camperdown, New South Wales, Australia

${ }^{3}$ Radiology Department, St Vincent's Hospital Sydney, Darlinghurst, New South Wales, Australia

${ }^{4}$ Clinic for Neurology, Heinrich Heine University Düsseldorf, Dusseldorf, Germany ${ }^{5}$ Neurology Department, Royal Prince Alfred Hospital, Camperdown, New South Wales, Australia

${ }^{6}$ Save Sight Institute, The University of Sydney, Sydney, New South Wales, Australia
}

Correction notice This article has been corrected since it appeared Online First. Equal and Senior authorship details have been added.

Contributors MHB, CW and AK conceived the study. CW and YB developed the imaging protocols. KK and LL performed MRI analysis. AK and JB developed the electrophysiological protocols and analysed the mFVEP and OCT data. MHB, SWR, $\mathrm{JB}, \mathrm{HB}$ and MT collected and analysed all clinical data. CW performed statistical testing. $\mathrm{H}-\mathrm{PH}$ and all authors made significant contributions to data analysis and interpretation, and drafting and revision of the manuscript.

Funding This study is funded by a Sanofi-Genzyme Research Grant: VisMS (Vision in MS: GZ-2015-11423). CW is supported by the Nerve Research Foundation, University of Sydney and Multiple Sclerosis Research Australia.

Competing interests CW has received research fellowship support from Multiple Sclerosis Research Australia, Nerve Research Foundation at the University of Sydney, and is an employee at Sydney Neuroimaging Analysis Centre (SNAC). JB has received honoraria for talks and advisory boards and support for scientific meetings from Sanofi-Genzyme, Novartis, Teva, Merck and Biogen. KK and LL are employees at SNAC. YB is a consulting neuroradiologist for SNAC. HB has received honoraria for presentations and advisory boards; and has received educational travel support from Biogen, Genzyme, Merck, Novartis and/or Roche. SWR has received institutional support from Baxter, Bayer Schering, Biogen Idec, CSL, Genzyme, Grifols, Octapharma, Merck, Novartis, Roche, Sanofi Aventis Genzyme, Servier and TEVA; and is a co-founder of Medical Safety Systems/RxMx. MHB reports grants from GenzymeSanofi, during the conduct of the study; grants from Genzyme-Sanofi, Novartis, Biogen and Merck outside the submitted work; and is a co-founder of RxMx, which provides automated laboratory monitoring for patients with MS prescribed immunotherapies; and research director of SNAC. H-PH has received fees for serving on steering and data monitoring committees from Bayer Healthcare, Biogen, Celgene BMS, CSL Behring, GeNeuro, Medlmmune, Merck, Novartis, Octapharma, Roche, Sanofi Genzyme, TG Therapeutics and Viela Bio; fees for serving on advisory boards from Biogen, Sanofi Genzyme, Merck, Novartis, Octapharma and Roche; and lecture fees from Biogen, Celgene BMS, Merck, Novartis, Roche and Sanofi Genzyme. MT and AK have nothing to disclose.

Patient consent for publication Not required.

Ethics approval The study was approved by the University of Sydney Human Research and Ethics Committee, and procedures adhered the tenets of the Declaration of Helsinki.

Provenance and peer review Not commissioned; externally peer reviewed.

Data availability statement All data relevant to the study are included in the article or uploaded as supplemental information.

Supplemental material This content has been supplied by the author(s). It has not been vetted by BMJ Publishing Group Limited (BMJ) and may not have been peer-reviewed. Any opinions or recommendations discussed are solely those of the author(s) and are not endorsed by BMJ. BMJ disclaims all liability and responsibility arising from any reliance placed on the content. Where the content includes any translated material, BMJ does not warrant the accuracy and reliability of the translations (including but not limited to local regulations, clinical guidelines, terminology, drug names and drug dosages), and is not responsible for any error and/or omissions arising from translation and adaptation or otherwise.

\section{ORCID iDs}

Chenyu Wang http://orcid.org/0000-0001-7135-7662

Michael Harry Barnett http://orcid.org/0000-0002-2156-8864

\section{REFERENCES}

1 Rocca MA, Mesaros S, Pagani E, et al. Thalamic damage and long-term progression of disability in multiple sclerosis. Radiology 2010;257:463-9.

2 Popescu V, Agosta F, Hulst HE, et al. Brain atrophy and lesion load predict long term disability in multiple sclerosis. J Neurol Neurosurg Psychiatry 2013;84:1082-91.

3 Tuohy O, Costelloe L, Hill-Cawthorne G, et al. Alemtuzumab treatment of multiple sclerosis: long-term safety and efficacy. J Neurol Neurosurg Psychiatry 2015;86:208-15.

4 Havrdova E, Arnold DL, Cohen JA, et al. Alemtuzumab CARE-MS I 5-year follow-up: durable efficacy in the absence of continuous MS therapy. Neurology 2017;89:1107-16.

5 Coles AJ, Cohen JA, Fox EJ, et al. Alemtuzumab CARE-MS II 5-year follow-up: efficacy and safety findings. Neurology 2017;89:1117-26.

6 Lassmann $\mathrm{H}$, Brück W, Lucchinetti C, et al. Remyelination in multiple sclerosis. Mult Scler 1997;3:133-6.

7 Prineas JW, Barnard RO, Kwon EE, et al. Multiple sclerosis: remyelination of nascent lesions: remyelination of nascent lesions. Ann Neurol 1993;33:137-51.

8 Liebetanz D, Merkler D. Effects of commissural de- and remyelination on motor skill behaviour in the cuprizone mouse model of multiple sclerosis. Exp Neurol 2006;202:217-24. 
9 Fancy SPJ, Kotter MR, Harrington EP, et al. Overcoming remyelination failure in multiple sclerosis and other myelin disorders. Exp Neurol 2010;225:18-23.

10 Barnett MH, Mathey E, Kiernan MC, et al. Axonal damage in central and peripheral nervous system inflammatory demyelinating diseases: common and divergent pathways of tissue damage. Curr Opin Neurol 2016;29:213-21.

11 De Stefano N, Airas L, Grigoriadis N, et al. Clinical relevance of brain volume measures in multiple sclerosis. CNS Drugs 2014;28:147-56.

12 Radue E-W, Barkhof F, Kappos L, et al. Correlation between brain volume loss and clinical and MRI outcomes in multiple sclerosis. Neurology 2015;84:784-93.

13 Barton JL, Garber JY, Klistorner A, et al. The electrophysiological assessment of visual function in multiple sclerosis. Clin Neurophysiol Pract 2019;4:90-6.

14 Cadavid D, Balcer L, Galetta S, et al. Safety and efficacy of opicinumab in acute optic neuritis (RENEW): a randomised, placebo-controlled, phase 2 trial. The Lancet Neurol 2017;16:189-99.

15 Hornabrook RSL, Miller DH, Newton MR, et al. Frequent involvement of the optic radiation in patients with acute isolated optic neuritis. Neurology 1992;42:77-9.

16 Alshowaeir D, Yiannikas C, Klistorner A. Multifocal visual evoked potential (mfVEP) and Pattern-Reversal visual evoked potential changes in patients with visual pathway disorders: a case series. Neuroophthalmology 2015;39:220-33.

17 Wang C, Klistorner A, Ly L, et al. White matter tract-specific quantitative analysis in multiple sclerosis: comparison of optic radiation reconstruction techniques. PLoS One 2018;13:e0191131.

18 Oh J, Ontaneda D, Azevedo C, et al. Imaging outcome measures of neuroprotection and repair in MS: a consensus statement from NAIMS. Neurology 2019;92:519-33

19 Mallik S, Samson RS, Wheeler-Kingshott CAM, et al. Imaging outcomes for trials of remyelination in multiple sclerosis. J Neurol Neurosurg Psychiatry 2014:85:1396-404.

20 Sormani MP, Pardini M. Assessing repair in multiple sclerosis: outcomes for phase II clinical trials. Neurotherapeutics 2017;14:924-33.

21 Klistorner A, Vootakuru N, Wang C, et al. Decoding diffusivity in multiple sclerosis: analysis of optic radiation lesional and non-lesional white matter. PLOS One 2015; 10:e0122114.

22 Song S-K, Sun S-W, Ramsbottom MJ, et al. Dysmyelination revealed through MRI as increased radial (but unchanged axial) diffusion of water. Neuroimage 2002;17:1429-36.

23 Klistorner A, Chai Y, Leocani L, et al. Assessment of Opicinumab in acute optic neuritis using multifocal visual evoked potential. CNS Drugs 2018;32:1159-71.
24 Klistorner A, Triplett JD, Barnett MH, et al. Latency of multifocal visual evoked potential in multiple sclerosis: a visual pathway biomarker for clinical trials of remyelinating therapies. J Clin Neurophysiol 2021;38:186-91.

25 Wang C, Barnett MH, Yiannikas C, et al. Lesion activity and chronic demyelination are the major determinants of brain atrophy in MS. Neurol Neuroimmunol Neuroinflamm 2019;6:e593.

26 Smith SM, Zhang Y, Jenkinson M, et al. Accurate, robust, and automated longitudinal and cross-sectional brain change analysis. Neuroimage 2002;17:479-89.

27 Battaglini M, Jenkinson M, De Stefano N. Evaluating and reducing the impact of white matter lesions on brain volume measurements. Hum Brain Mapp 2012;33:2062-71.

28 Barton J, Hardy TA, Riminton S, et al. Tumefactive demyelination following treatment for relapsing multiple sclerosis with alemtuzumab. Neurology 2017;88:1004-6.

29 Ziemssen T, Bass AD, Berkovich R, et al. Efficacy and safety of alemtuzumab through 9 years of follow-up in patients with highly active disease: post hoc analysis of CAREMS I and II patients in the Topaz extension study. CNS Drugs 2020;34:973-88.

30 Green AJ, Gelfand JM, Cree BA, et al. Clemastine fumarate as a remyelinating therapy for multiple sclerosis (ReBUILD): a randomised, controlled, double-blind, crossover trial. Lancet 2017;390:2481-9.

31 Gingele S, Stangel M. Emerging myelin repair agents in preclinical and early clinical development for the treatment of multiple sclerosis. Expert Opin Investig Drugs 2020;29:583-94

32 Wiendl H, Bourdette D, Ciccarelli O. Can immune reprogramming with alemtuzumab induce permanent remission in multiple sclerosis? Neurology 2017;89:1098-100

33 Klistorner A, Wang C, Yiannikas C, et al. Evidence of progressive tissue loss in the core of chronic MS lesions: a longitudinal DTI study. Neuroimage 2018;17:1028-35.

34 Wang CT, Barnett M, Barnett Y. Imaging the multiple sclerosis lesion: insights into pathogenesis, progression and repair. Curr Opin Neurol 2019;32:338-45.

35 Lema A, Bishop C, Malik 0, et al. A comparison of magnetization transfer methods to assess brain and cervical cord microstructure in multiple sclerosis. J Neuroimaging 2017;27:221-6.

36 Prasloski T, Rauscher A, MacKay AL, et al. Rapid whole cerebrum myelin water imaging using a 3D GRASE sequence. Neuroimage 2012;63:533-9.

37 Arshad M, Stanley JA, Raz N. Test-retest reliability and concurrent validity of in vivo myelin content indices: Myelin water fraction and calibrated $T_{1} w / T_{2} w$ image ratio. Hum Brain Mapp 2017;38:1780-90.

38 Saccenti L, Hagiwara A, Andica C, et al. Myelin measurement using quantitative magnetic resonance imaging: a correlation study comparing various imaging techniques in patients with multiple sclerosis. Cells 2020;9:393. 